\title{
Bilateral Posterior Periventricular Nodular Heterotopia: A Recognizable Cortical Malformation with a Spectrum of Associated Brain Abnormalities
}

S.A. Mandelstam, R.J. Leventer, A. Sandow, G. McGillivray, M. van Kogelenberg, R. Guerrini, S. Robertson, S.F. Berkovic, G.D. Jackson, and I.E. Scheffer

\begin{abstract}
BACKGROUND AND PURPOSE: Bilateral posterior PNH is a distinctive complex malformation with imaging features distinguishing it from classic bilateral PNH associated with FLNA mutations. The purpose of this study was to define the imaging features of posterior bilateral periventricular nodular heterotopia and to determine whether associated brain malformations suggest specific subcategories.
\end{abstract}

MATERIALS AND METHODS: We identified a cohort of 50 patients (31 females; mean age, 13 years) with bilateral posterior PNH and systematically reviewed and documented associated MR imaging abnormalities. Patients were negative for mutations of FLNA.

RESULTS: Nodules were often noncontiguous $(n=28)$ and asymmetric $(n=31)$. All except 1 patient showed associated developmental brain abnormalities involving a spectrum of posterior structures. A range of posterior fossa abnormalities affected the cerebellum, including cerebellar malformations and posterior fossa cysts $(n=38)$. Corpus callosum abnormalities $(n=40)$ ranged from mild dysplasia to agenesis. Posterior white matter volume was decreased $(n=22)$, and colpocephaly was frequent $(n=26)$. Most $(n=40)$ had associated cortical abnormalities ranging from minor to major (polymicrogyria), typically located in the cortex overlying the PNH. Abnormal Sylvian fissure morphology was common $(n=27)$, and hippocampal abnormalities were frequent $(n=37)$. Four family cases were identified-2 with concordant malformation patterns and 2 with discordant malformation patterns.

CONCLUSIONS: The associations of bilateral posterior PNH encompass a range of abnormalities involving brain structures inferior to the Sylvian fissures. We were unable to identify specific subgroups and therefore conceptualize bilateral posterior PNH as a continuum of infrasylvian malformations involving the posterior cerebral and hindbrain structures.

ABBREVIATIONS: DHPLC = denaturing high-performance liquid chromatography; FLNA = Filamin A gene; PNH = periventricular nodular heterotopia

$\mathbf{S}$ ubependymal or periventricular nodular heterotopia is the most commonly identified gray matter heterotopia in clinical practice. ${ }^{1-5}$ It may be more often associated with other malformations than commonly appreciated. ${ }^{5} \mathrm{PNH}$ is a collection of sub-

Received February 29, 2012; accepted after revision April 17.

From the Florey Neurosciences Institute (S.A.M., G.D.J., I.E.S.), Melbourne, Australia; Departments of Radiology (S.A.M.) and Neurology (R.J.L., I.E.S.), Royal Children's Hospital and Department of Paediatrics (R.J.L., I.E.S.), University of Melbourne, Melbourne, Australia; Epilepsy Research Centre (A.S., S.F.B., I.E.S.), Department of Medicine, University of Melbourne, Austin Health, Melbourne, Australia; Murdoch Childrens Research Institute (R.J.L., G.M.), Melbourne, Australia; Clinical Genetics Group (M.v.K., S.R.), Dunedin School of Medicine, University of Otago, Dunedin, New Zealand; and Division of Child Neurology and Psychiatry (R.G.), University of Pisa and IRCCS Fondazione Stella Maris, Pisa, Italy.

This paper was funded by a grant from the National Health and Research Council of Australia.

Please address correspondence to Graeme Jackson, MD, Florey Neuroscience Institutes, Austin, Melbourne Brain Centre, 245 Burgundy St, Heidelberg, 3084, Victoria, Australia: e-mail: g.jackson@brain.org.au

- Indicates open access to non-subscribers at www.ajnr.org

三 Indicates article with supplemental on-line tables.

http://dx.doi.org/10.3174/ajnr.A3427 ependymal nodules of gray matter found along the walls of the lateral ventricles. ${ }^{1,6-9} \mathrm{PNH}$ consists of clusters of neurons that fail to migrate away from the embryonic ventricular zone to the developing cerebral cortex. Radial migration occurs maximally between the seventh and sixteenth gestational weeks, along radial glial fibers. ${ }^{10-13}$ The major locus associated with PNH is the Filamin A gene on Xq28 that causes X-linked $\mathrm{PNH}$ that is typically bilateral and symmetric. This classic form occurs in females, many of whom have normal intelligence and epilepsy. ${ }^{14}$ The nodules in these patients involve predominantly the anterior bodies and frontal horns of the lateral ventricles and are often diffuse, contiguous, and symmetric. ${ }^{9,15-17}$

Our study was motivated by the observation that the second most common pattern of PNH showed nodules predominantly along the atria and temporal horns of the lateral ventricles. Patients with this posterior form of $\mathrm{PNH}$ were FLNA-negative, had symptoms that appeared different from those in the classic form, and were a sizeable group that were underappreciated as a separate entity in the literature. Reported subtypes of FLNA-negative 
PNH include PNH with hydrocephalus ${ }^{10,18}$; PNH with microcephaly ${ }^{10,18}$; $\mathrm{PNH}$ with frontonasal dysplasia ${ }^{8}$; $\mathrm{PNH}$ with limb abnormalities ${ }^{14}$; PNH with fragile $\mathrm{X}$ syndrome; $\mathrm{PNH}$ with ambiguous genitalia; and, micronodular, unilateral, laminar, ribbonlike, and linear $\mathrm{PNH} .{ }^{9} \mathrm{We}$ also observed that patients with posterior $\mathrm{PNH}$ had more frequent abnormalities of other brain structures compared with patients with classic bilateral PNH. We therefore questioned whether bilateral posterior PNH is a distinct entity compared with classic $\mathrm{PNH}$ and reviewed all cases in which the nodules were primarily posterior and bilateral. We examined the associated brain abnormalities to determine whether there were subcategories within the posterior $\mathrm{PNH}$ group or whether posterior PNH is part of a spectrum of disordered infra-Sylvian development.

\section{MATERIALS AND METHODS \\ Imaging}

We identified 50 patients with posterior-predominant $\mathrm{PNH}$ on MR imaging scans referred by multiple centers. Structural MR imaging examinations were performed between 1996 and 2009. All patients had multiplanar multiecho sequences, including T1, T2, and FLAIR. T1 images were of high quality, typically by using an inversion-prepared sequence, and were of appropriate section thickness, typically 3 $\mathrm{mm}$. T1- and T2-weighted images were available in all cases.

We classified each patient's MR images on the basis of imaging findings. The shape, location, and symmetry of PNH were analyzed, and the presence of all associated abnormalities was documented. Apart from describing the features of the PNH, we assessed associated brain structures as follows:

1) Cerebellum: morphology of the vermis and hemispheres

2) Posterior fossa: presence or absence of a posterior fossa cyst

3) Brain stem morphology

4) Corpus callosum: the presence and shape of the corpus callosum

5) White matter: volume and signal intensity

6) Ventricles: morphology and size of the ventricles. Lateral ventricles were assessed for colpocephaly, defined as disproportionate dilation of the trigones and occipital horns of the lateral ventricles. $^{19}$

7) Hippocampi: symmetry, shape, and signal intensity of the hippocampi. Hippocampi were assessed as asymmetric if the shape or size of the 2 sides differed but the internal architecture was preserved with normal signal intensity and normal hippocampal rotation. Dysplasia was assessed by using the following criteria: shape, size, signal intensity, and hippocampal rotation.

8) Cerebral cortex: The cortical mantle was assessed for the presence of cortical malformations such as cortical dysplasia and polymicrogyria.

\section{Filamin A Analysis}

FLNA was examined by either DHPLC (Transgenomic, Omaha, Nebraska) or Sanger sequencing by using BigDye ter- minator chemistry (http://products.invitrogen.com/ivgn/product/ 433745) and was analyzed on an ABI3100 automated sequencer (Applied Biosystems, Foster City, California), by using genomic DNA samples obtained from each patient. Polymerase chain reaction primers and conditions were previously described. ${ }^{20}$ For male subjects undergoing denaturing high-performance liquid chromatography analysis, amplified DNA was mixed with an equal amount of polymerase chain reaction product from an unrelated male control individual who had been sequence-verified to have no sequence variants in the amplicon under study. Anomalous waveforms obtained during denaturing high-performance liquid chromatography analysis were Sanger-sequenced on re-amplified DNA. Additionally 8 intragenic (intron 2, exons 6, 15, 22, 29, 34, 44, and 48) and 4 flanking (GDI1, TKTL1, EMD, and MECP2) sites were evaluated by using a custom-designed multiplex ligation-dependent probe amplification assay kit to ascertain for duplications or deletions over the $26 \mathrm{~kb}$ FLNA locus by using standard experimental and analytic protocols as specified by the manufacturer (MRC-Holland, Amsterdam, the Netherlands).

The study was approved by the human research ethics committees at Austin Heath and the Royal Children's Hospital, Melbourne.

\section{RESULTS}

\section{Patient Characteristics}

The 50 patients ranged in age from a 26-week fetus (in utero scan) to 67 years of age, with a mean postnatal age of 13 years and a median age of 6 years at the time of the first MR imaging. There were 31 females (62\%). Forty-two were unrelated individuals. There were 2 sister pairs, 1 monozygotic twin brother pair, and 1 mother-son pair. The son was imaged as a fetus with 2 fetal MR imaging scans at 26 weeks' and 32 weeks' gestation as well as with a postnatal study.

\section{Nodules}

We defined posterior $\mathrm{PNH}$ as either restricted to or predominant in the region around the posterior horns, atria, or temporal horns of the lateral ventricles. Heterotopic nodules had an exclusively posterior infrasylvian distribution in $35(70 \%)$ patients, while 15 (30\%) had a mixed picture with a predominance of posterior nodules and scattered nodules anteriorly along the bodies of anterior horns. Nodules were contiguous in $22(44 \%)$ patients and symmetric in $19(38 \%)$ patients. 

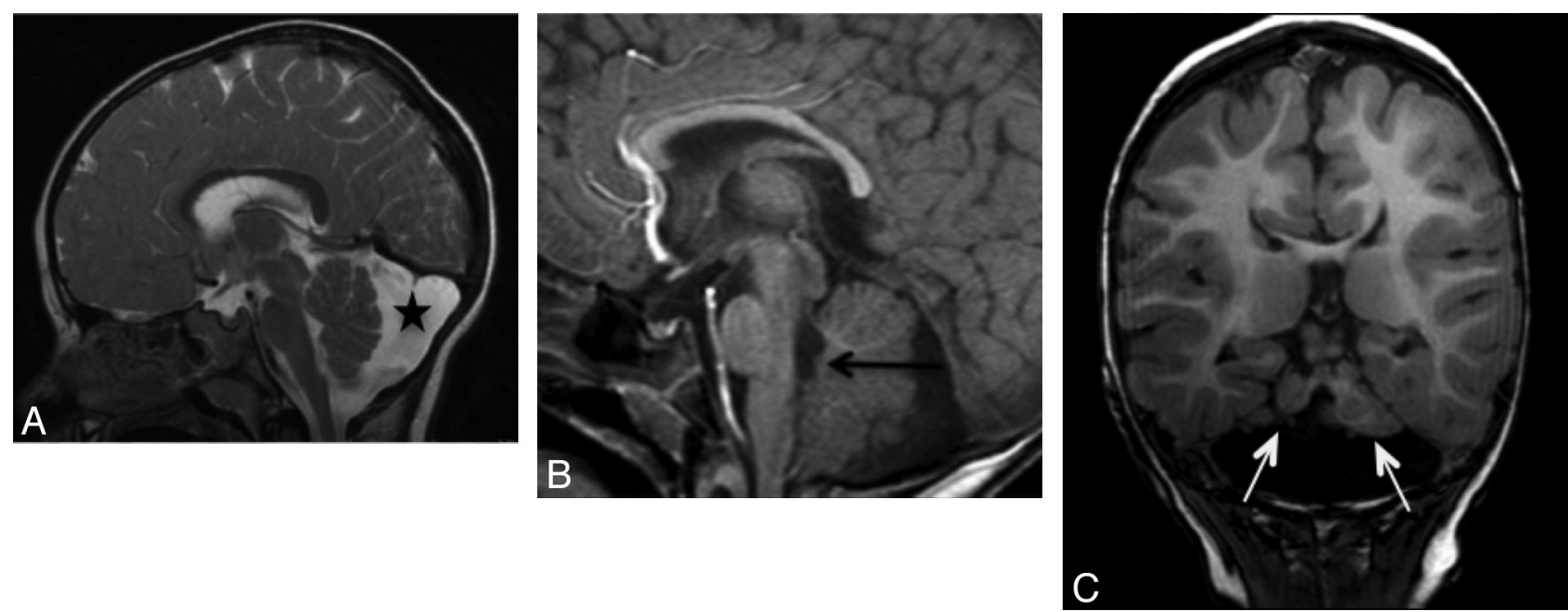

FIG 2. Examples of 3 different posterior fossa abnormalities. A, Sagittal T2 image demonstrates a morphologically normal but small cerebellum with a large retrocerebellar cyst (star). B, Sagittal $T 1$ image demonstrates a bulky cerebellar vermis with a nodule projecting into the fourth ventricle (arrow). C, Coronal $\mathrm{Tl}$ image of a malformed cerebellum with asymmetric hypoplasia of both cerebellar hemispheres (arrows).

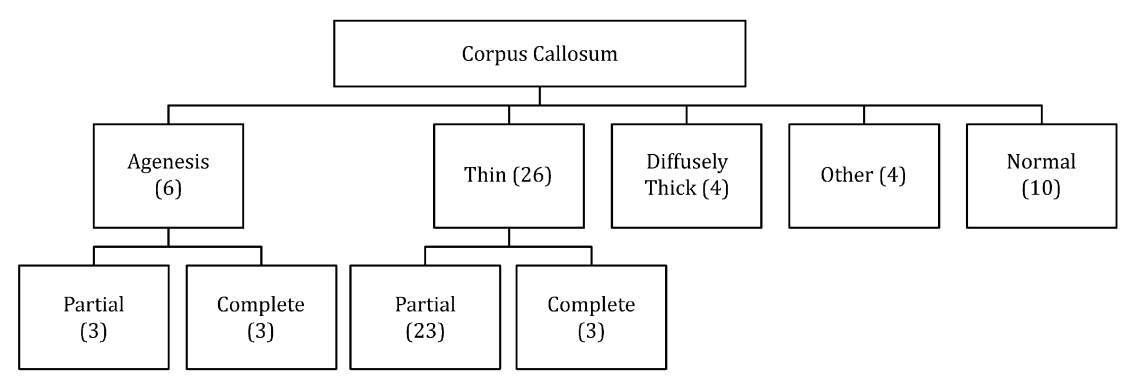

FIG 3. Spectrum of the corpus callosum appearance in the posterior $\mathrm{PNH}$.

major groups: agenesis (6 patients), thin (21 patients), thick ( 4 patients), and minor irregularities (4 patients), (Figs 3 and 4).

All patients had abnormalities of the whole or the posterior half of the corpus callosum. There were 3 patients with complete agenesis of the corpus callosum and 3 with partial agenesis who had absence of the posterior body and splenium. Five of these patients had abnormal hippocampi. Twenty-three (46\%) patients had

\section{Posterior Fossa Abnormalities}

Thirty-eight of $50(76 \%)$ patients had a range of posterior fossa abnormalities (Fig 1). The major abnormalities included severe dysplasia/hypoplasia of the cerebellar hemispheres with large CSF fluid collections in the Dandy-Walker continuum. Twenty of 50 (40\%) patients had dysplastic cerebella with vermian abnormalities. Seventeen patients with cerebellar abnormalities had posterior fossa cysts. The appearance of the cysts varied from small unilateral cysts with no mass effect to large cysts expanding the posterior fossa. The latter were associated with extremely small and malformed cerebellar hemispheres. Minor abnormalities included deficiency of the tentorium cerebelli. Five patients had small but morphologically normal cerebella with posterior fossa cysts (Fig 2).

With 2 exceptions, all patients with cerebellar abnormalities also had abnormalities of the corpus callosum and were more likely to have white matter abnormalities. Ten patients (20\%) had brain stem abnormalities, including a patient with a tectal plate glioma and 6 patients with tectal plate thickening. The other 3 had unilateral or bilateral brain stem hypoplasia.

\section{Corpus Callosum and White Matter}

Forty of $50(80 \%)$ patients demonstrated abnormalities of the corpus callosum ranging from mild dysplasia to complete agenesis. The corpus callosum abnormalities were divided into 1 of 4 thin posterior bodies and splenia of the corpus callosum with normal anterior components, while 3 patients had thinning of the entire corpus callosum. Four patients had an unusual thickened appearance of the corpus callosum with dysplastic splenia and no colpocephaly. All 4 had associated hippocampal dysplasia and abnormal Sylvian fissures.

Fifteen patients with callosal abnormalities had a normal cerebellum, while 7 patients had a normal cerebellum and a normal corpus callosum. These patients were more likely to have hippocampal abnormalities and cortical/sulcation abnormalities, ranging from Sylvian fissure abnormalities to areas of cortical thickening and suspected transmantle bands.

Twenty-two (44\%) patients demonstrated decreased posterior white matter volume, and 5 patients had nonspecific increased T2 signal intensity within the white matter. Two of these had unilateral anterior temporal white matter signal abnormality of the type seen in temporal lobe dysplasias or hippocampal sclerosis. One patient demonstrated delayed myelination for age, and 1 had white matter distortion due to multiple nodules.

\section{Ventricles}

Thirty-two (64\%) patients had ventricular abnormalities. Of these, 26 patients had colpocephaly. In 3 cases, the ventricle outlines were very distorted by the presence of the nodules. There was mild generalized ventriculomegaly in 3 patients. 

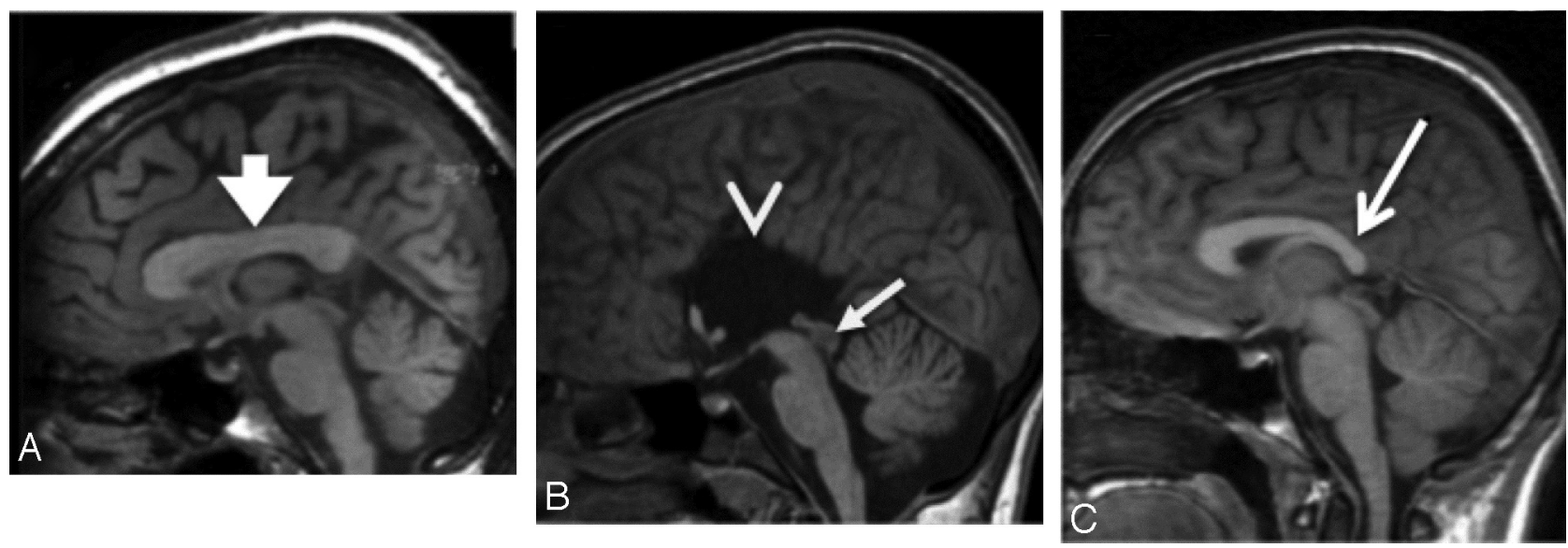

FIG 4. Examples of 3 different corpus callosum abnormalities. A, Thick corpus callosum (arrow). B, Agenesis of the corpus callosum (arrowhead). Note tectal glioma (arrow). C, Thinning of the posterior body and splenium (arrow).

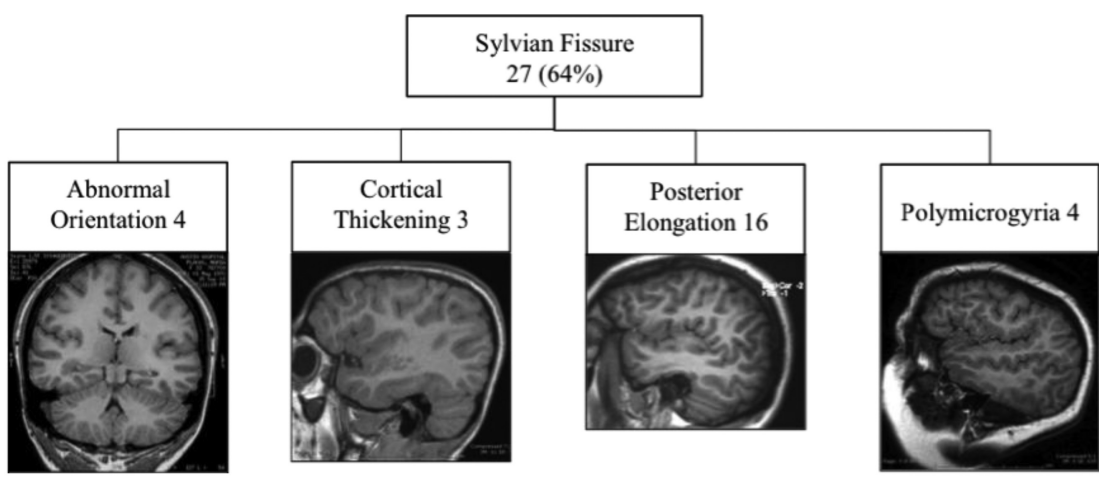

an occipital encephalocoele repaired as an infant with associated venous anomalies. One patient had lobar holoprosencephaly. A pair of female siblings had congenital cataracts with optic nerve hypoplasia.

\section{Familial Cases}

There were 4 families with 2 affected individuals in each family (2 sibling pairs, 1 pair of monozygotic twins, and a motherson pair). The first sister pair and the mother-son pair showed diverse malformations, while the twins and the second sister pair had concordant malformations

\section{Cortical and Sulcation Abnormalities}

Forty $(80 \%)$ patients had associated cortical mantle or sulcation abnormalities, usually in the cortex directly overlying the PNH. These included nonspecific cortical thickening, linear transmantle bands, cortical dysplasia, and polymicrogyria (On-line Table 1). Linear transmantle bands were T1 hypointense and T2 hyperintense lines extending from the nodules to the overlying cortical surface. Twelve of 50 (24\%) had cortical dysplasia, schizencephaly, or polymicrogyria. There were 11 patients with sulcation abnormalities alone; 4 with transmantle bands alone; and 13 with combinations of cortical thickening, linear transmantle bands, and sulcation abnormalities. Twenty-seven (54\%) patients had abnormal Sylvian fissures with a spectrum of appearances as shown in Fig 5.

\section{Hippocampal Abnormalities}

There was a spectrum of hippocampal abnormalities in $37 / 50$ (74\%) patients. Twenty-five patients had dysplastic hippocampi, of which 16 were also malrotated. Twelve (24\%) had asymmetric hippocampi (Fig 6).

\section{Additional Abnormalities}

Five patients had abnormalities of the pituitary gland. These included an absent posterior pituitary hyperintense neurohypophysis ("bright spot") $(n=1)$, an ectopic posterior pituitary hyperintense neurohypophysis $(n=2)$, a pituitary cyst $(n=1)$, and a duplicated pituitary hyperintense region $(n=1)$. One patient had

(Fig 7 and On-line Tables 1 and 2).

\section{Filamin A Analysis}

Examination of all coding exons and intron-exon boundaries of FLNA was completed for 40 subjects. No point mutations or small insertion/deletions confined to a single exon were detected in any subject. Additionally, no deletions or duplications of either the entire locus or involving the assayed exons were detected. Thus there were no sequencing mutations or copy number variants of FLNA found in the tested cohort with bilateral posterior PNH.

\section{DISCUSSION}

Bilateral posterior $\mathrm{PNH}$ is a cortical malformation entity showing a spectrum of disordered brain development and is distinct from classic bilateral PNH due to not only the location of the nodules but also to the common occurrence of malformations of other brain structures and the absence of mutations in the FLNA gene. We found only 1 case that had no identifiable malformations other than the PNH. This is despite the selection criteria being only bilateral posterior nodules, admittedly in the context of a tertiary referral center. In some cases these abnormalities were only detected in this systematic review and were not reported on the routine studies that led to recruitment. We suggest, therefore, that posterior bilateral PNH is typically associated with other brain malformations and that it is part of a more diffuse process of posterior or infrasylvian brain maldevelopment other than just a disorder of neuronal migration.

AJNR Am J Neuroradiol 34:432-38 Feb 2013 www.ajnr.org 


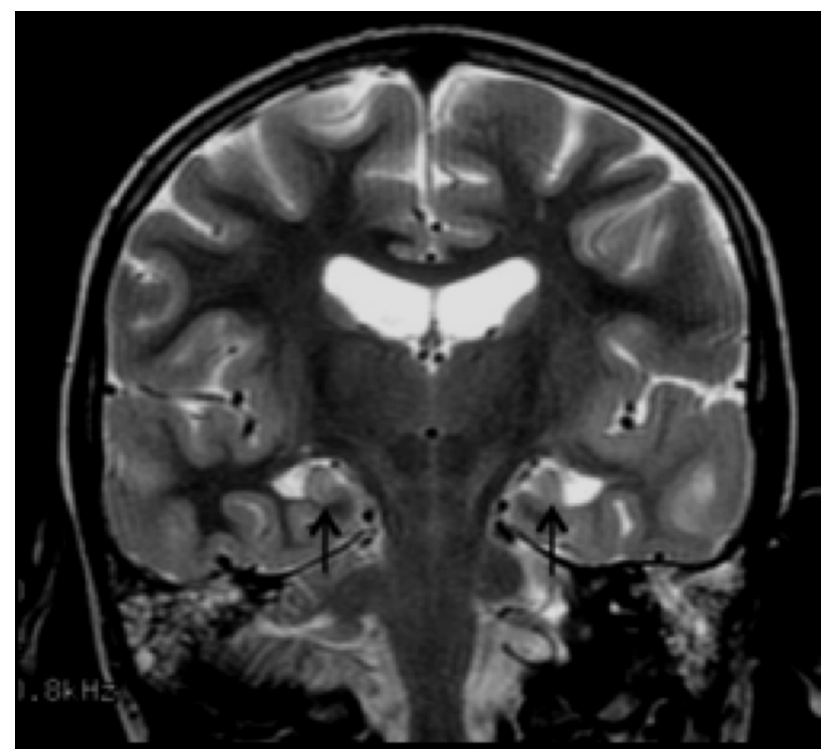

FIG 6. Coronal $\mathrm{T} 2$ image demonstrating dysplastic and under-rotated hippocampi (arrows).

\section{Associated Malformations}

Cortical abnormalities occurred in $80 \%$ of cases, usually in the cortex overlying the PNH. Sylvian fissures were often abnormal (50\%), some demonstrating minor anomalies such as elongation and some with severe abnormalities such as bilateral perisylvian polymicrogyria. These could be secondary to a disturbance in a range of fundamental forces shaping the cortex, including processes underlying the normal gyrification of the brain, such as genetic control of sulcation, cytoarchitecture, differential cortical growth, and white matter tension morphogenesis. ${ }^{21-28}$

Posterior callosal abnormalities were found in $80 \%$ of patients. This was typically thinning or agenesis and likely reflects disordered or failed migration or defective axon guidance. Although the recognized association of callosal and hippocampal
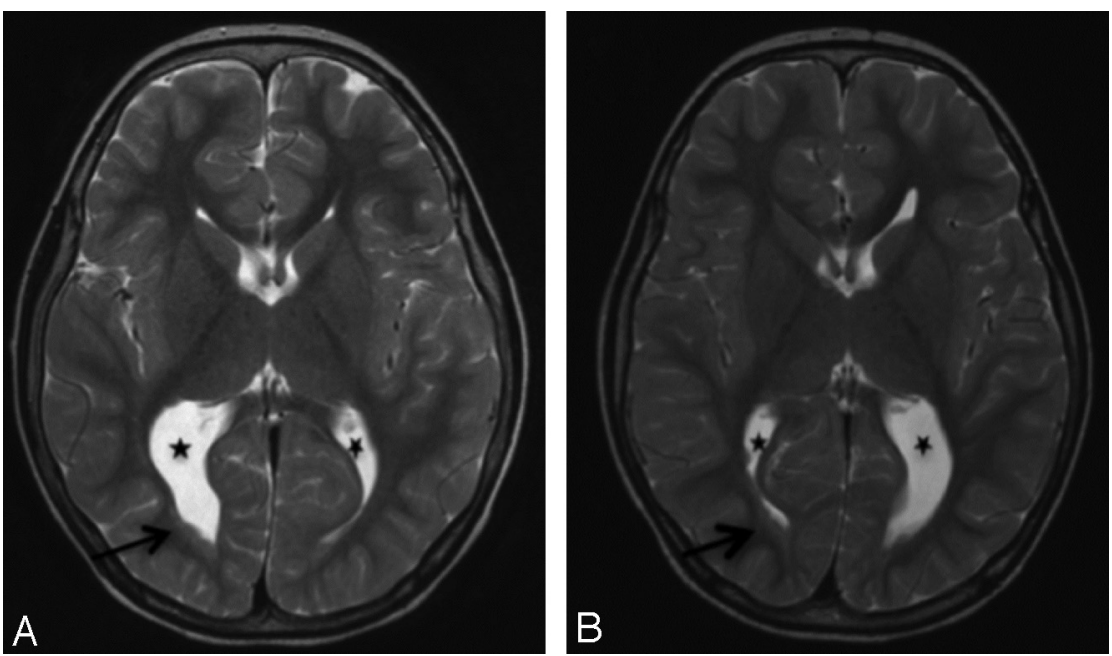

FIG 7. Images of twin sibling pair 1 (Fig $1 A,-B$ ). Comparable axial T2 images of 5-year-old monozygotic twin boys demonstrate almost mirror-image appearances with asymmetric colpocephaly (stars) and nodules lining the posterior horns right $>$ left (arrows). Other features (not shown) were small cerebella, small posterior fossa cysts, and thinned posterior white matter including the posterior corpus callosum. Cortical and sulcation abnormalities differed between them. abnormalities was seen in our patients, we did not see this combination as a separate subgroup. ${ }^{1,3,29-31}$ The arrested hippocampal rotation is likely to be due to secondary effects of abnormal development, though we acknowledge it could be part of the same genetic defect that arrests the migration of neural progenitor cells. ${ }^{32}$ Four patients had callosal hypertrophy with dysplastic splenia, hippocampal dysplasia, and abnormal Sylvian fissures. A hyperplastic corpus callosum is rare and has been reported in neurofibromatosis type $1^{33}$ and Cohen syndrome ${ }^{34,35}$ and likely reflects a failure of normal pruning of the commissural fibers. ${ }^{36,37}$

We commonly saw posterior ventricular dilation (50\%), which we suspect has sometimes been interpreted as hydrocephalus. ${ }^{9}$ Our interpretation is that this is "colpocephaly," because our cases had no clinical or other imaging features suggesting raised intracranial pressure.

Cerebellar abnormalities of varying severity were found in $50 \%$ of patients and varied from asymmetric cerebellar hemisphere hypoplasia, universally associated with a small or absent vermis, to a "microcerebellum" that was small but normally formed. A bulky vermis was seen in 5 patients, likely due to nodular heterotopia causing expansion. One patient had a small tectal plate lesion that mimicked gray matter on all sequences and did not enhance, suggesting a periventricular nodule rather than an indolent midbrain tumor. ${ }^{38}$ Previously reported pituitary gland abnormalities were seen in $10 \%$ of patients, including one with an ectopic posterior pituitary gland. ${ }^{39}$

\section{Genetics of the Bilateral Posterior PNH Spectrum}

At least some bilateral infra-Sylvian PNH has a genetic basis as demonstrated by our 4 kinships that include identical twins and a mother-son pair. Each related pair of individuals showed a range of abnormalities within the bilateral posterior PNH "spectrum." This is strong clinical evidence of a genetic basis underlying critical aspects of this developmental disorder. The twins and second sister pair were concordant with respect to associated malformations within each pair. The first sister pair and the mother-son pair showed variation in their malformations. There were no clear environmental or acquired factors to explain the disparity in the related pairs. These differences with presumed common genes, best illustrated by the mother-son pair, indicate that there may be a spectrum of expressions of common mutations or modifying factors. This suggests that common pathways may be involved in the range of features we see in this bilateral PNH cohort.

Bilateral posterior $\mathrm{PNH}$ is a distinct malformation of cortical development associated with a spectrum of infrasylvian developmental abnormalities. The fact that we could identify a series of 50 such cases suggests that posterior $\mathrm{PNH}$ is a large subgroup of the common bilateral PNH cases. In our experience of $\sim 200$ patients with bilateral $\mathrm{PNH}$, it is the second 


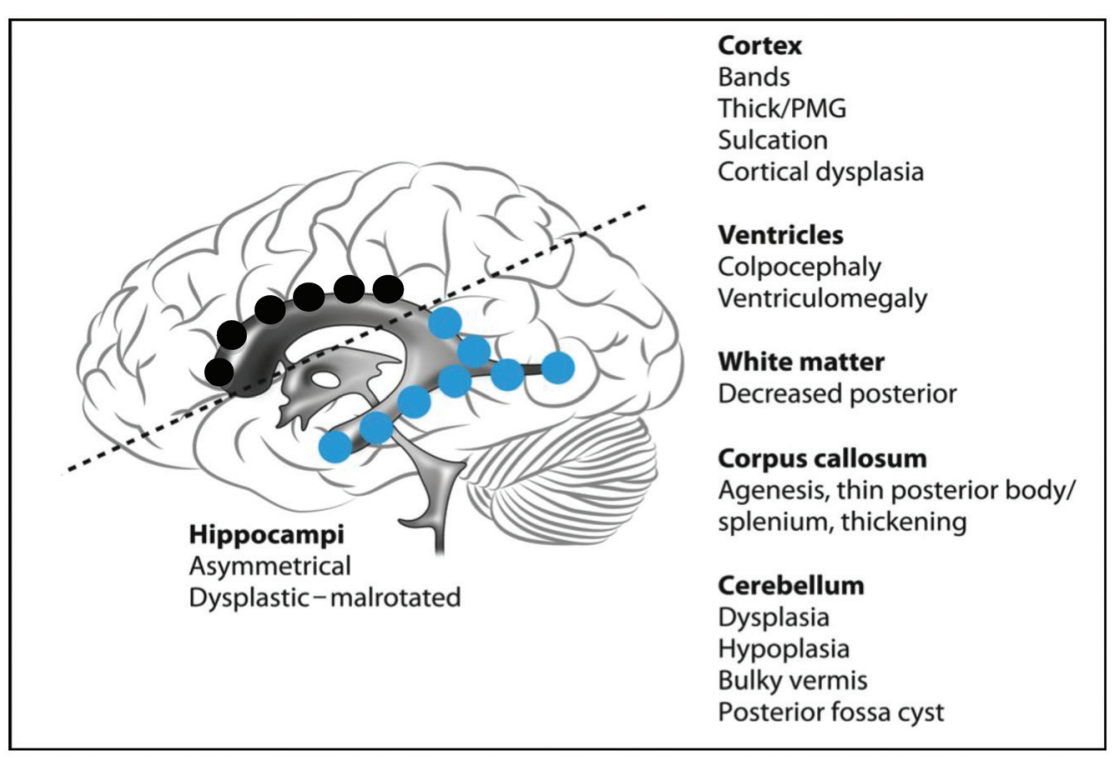

FIG 8. Summary of the malformation spectrum seen in bilateral posterior $\mathrm{PNH}$, highlighting the infrasylvian location of the nodules and the associated malformations. Black nodules represent typical frontocentral nodules and blue nodules represent infrasylvian location.

usually isolated or also associated with developmental malformations.

\section{Connectivity}

While periventricular nodules have been shown in pathologic studies to have mostly sparse connections with extranodular regions and with other adjacent nodules, some nodules have been shown to be more connected to overlying and potentially abnormal cortex than others. ${ }^{42,43}$ Epileptic activity may be due to a combination of the intrinsic epileptogenicity of the nodule as well as its connections to cortex. ${ }^{42-44}$

\section{CONCLUSIONS}

In nonsyndromic bilateral posterior $\mathrm{PNH}$, Parrini et $\mathrm{al}^{9}$ distinguished 3 major associations: hippocampal malformation and cerebellar hypoplasia $(n=10)$, polymicrogyria $(n=2)$, and hydrocephalus $(n=5)$. We selected patients who had similar PNH features but could not easily fit them into these

most common pattern after classic frontocentral $\mathrm{PNH}$ and accounts for $\sim 25 \%$ of all cases. Classic PNH has been the subject of extensive radiologic and molecular investigation with a clear definition of the frontocentral location of the $\mathrm{PNH}$, which is often symmetric, and a strong association with FLNA mutations. ${ }^{17,40,41}$ Here we differentiate posterior $\mathrm{PNH}$ on the basis of imaging and molecular abnormalities because none of the 40 cases tested had FLNA mutations.

While the term "posterior" has been used to describe our cases, in common anatomic and radiologic practice, many of our cases would be described as anterior, particularly with nodules in the anterior temporal poles. To deal with this difference, the term "temporo-trigonal" has been proposed. We believe even this is a limited anatomic description, and we would prefer to divide the 2 major forms of bilateral PNH into "suprasylvian" and "infrasylvian" to accord more accurately with the distribution of the nodules. We leave this suggestion for future discussions as these entities become better characterized. It will be important to review this concept of associated malformations in other cohorts of bilateral posterior $\mathrm{PNH}$.

This is an important concept because, unlike the classic PNH, $98 \%$ of patients with bilateral posterior $\mathrm{PNH}$ have associated congenital brain malformations, suggesting that this $\mathrm{PNH}$ is a marker of a more extensive disturbance in cortical development and possibly reflects aberrations in a different mechanism or pathway to classic PNH. It is interesting to speculate why only structures inferior to the Sylvian fissure are involved, with $>74 \%$ demonstrating posterior fossa, posterior corpus callosum, hippocampal, and posterior cortical abnormalities alone or in combination. These are the developmental areas associated with the location of the nodules, but why only these regions are affected challenges our understanding of embryology and "posterior" brain development. Unilateral nodules were not addressed in this article, and it will be of future interest to determine whether they are
3 specific categories. Rather than discrete subgroups, we suggest that bilateral posterior $\mathrm{PNH}$ is a distinct malformation entity that encompasses a continuum of brain malformations involving posterior or infrasylvian structures (Fig 8).

Disclosures: George McGillivray—UNRELATED: Employment: Victorian Clinical Genetics Services (Melbourne), Comments: I am employed full-time as a clinical geneticist providing clinical diagnostic services. Renzo Guerrini-UNRELATED: Board Membership: Biocodex, UCB Pharma, Eisai Pharmaceuticals, ViroPharma Box, European Medicine Agency, Consultant: EISAI; Royalties: Epilepsy and Movements Disorders, Cambridge University Press, 2002; Aicardi's Epilepsy in Children, Lippincott Williams \& Wilkins, 2004; Progress in Epileptic Spasms and West Syndrome, John Libbey Eurotext, 2007; Epilepsy and Migraine, John Libbey Eurotext 2009; The Causes of Epilepsy, Cambridge University Press, 2011, Travel/Accommodations/ Meeting Expenses Unrelated to Activities Listed: Biocodex, Eisai Pharmaceuticals, Japanese Epilepsy Society, Weill Cornell Medical College in Qatar. Samuel E. Berkovic-RELATED: Grant: National Health and Clinical Research Council.* Ingrid E. Scheffer-UNRELATED: Payment for Lectures (including service on Speakers Bureaus): UCB Pharma, Janssen-Cilag, Athena Diagnostics, Biocodex, Comments: UCB: Speaker 2009, 2011; Janssen-Cilag: Speaker, 2009; Athena Diagnostics: Speaker, 2009; Biocodex: Speaker, 2012, Patents (planned, pending, or issued): Pending patent: WO61/010176, Therapeutic Compound (filed 2008), ${ }^{*}$ Payment for Development of Educational Presentations: Athena Diagnostics, Comments: 2009, 2010, Travel/Accommodations/Meeting Expenses Unrelated to Activities Listed: UCB Pharma, Biocodex, Athena Diagnostics, GlaxoSmithKline, Comments: UCB: Keppra Paediatric Epilepsy Summit, 2009; Symposium, Korea, 2010; Australian Neurology Update, 2011; 45th Annual Congress of the Japan Epilepsy Society, 2011; Biocodex: International Epilepsy Congress Satellite Symposium, 2011; Athena Diagnostics: American Epilepsy Society Symposium, 2009; GlaxoSmithKline: Japanese Epilepsy Society Meeting, 2012. *Money paid to the institution.

\section{REFERENCES}

1. Aghakhani Y, Kinay D, Gotman J, et al. The role of periventricular nodular heterotopia in epileptogenesis. Brain 2005;128:641-51

2. Barkovich AJ, Kjos BO. Gray matter heterotopia: MR characteristics and correlation with developmental and neurologic manifestations. Radiology 1992;182:493-99

3. Raymond AA, Fish DR, Stevens JM, et al. Subependymal heterotopia: a distinct neuronal migration disorder associated with epilepsy. J Neurol Neurosurg Psychiatry 1994;57:1195-202

4. Dubeau F, Tampieri D, Lee N, et al. Periventricular and subcortical nodular heterotopia: a study of 33 patients. Brain 1995;118:1273-87 
5. Li LM, Dubeau F, Andermann F, et al. Periventricular nodular heterotopia and intractable temporal lobe epilepsy: poor outcome after temporal lobe resection. Ann Neurol 1997;41:662-68

6. Wieck G, Leventer R, Squier W, et al. Periventricular nodular heterotopia with overlying polymicrogyria. Brain 2005;128:2811-21

7. Guerrini R. Genetic malformations of the cerebral cortex and epilepsy. Epilepsia 2005;46(suppl 1):32-37

8. Guerrini R, Dobyns W. Bilateral periventricular nodular heterotopia with mental retardation and frontonasal malformation. Neurology 1998;51:499-503

9. Parrini E, Ramazzotti A, Dobyns WB, et al. Periventricular heterotopia: phenotypic heterogeneity and correlation with Filamin A mutations. Brain 2006;129:1892-906

10. Caviness VS Jr, Rakic P. Mechanisms of cortical development: a view from mutations in mice. Annu Rev Neurosci 1978;1:297-326

11. Rakic $P$. The cell in contact: adhesions and junctions as morphogenetic determinant. In: Edelman G, Thiery J, eds. Contact Regulation of Neuronal Migration. New York: Neuroscience Research Foundation; 1985:67-91

12. Rakic P. Radial versus tangential migration of neuronal clones in the developing cerebral cortex. Proc Natl Acad Sci U S A 1995;92: 11323-27

13. Sarnat HB. Cerebral dysplasias as expressions of altered maturational processes. Can J Neurol Sci 1991;18:196-204

14. Dobyns WB, Guerrini R, Czapansky-Beilman DK, et al. Bilateral periventricular nodular heterotopia with mental retardation and syndactly in boys: a new X-linked mental retardation syndrome. Neurology 1997;49:1042-47

15. Poussaint TY, Fox JW, Dobyns WB, et al. Periventricular nodular heterotopia in patients with filamin-1 gene mutations: neuroimaging findings. Pediatr Radiol 2000;30:748-55

16. Ekşioğlu YZ, Scheffer I, Cardenas P. Periventricular heterotopia: an $\mathrm{X}$-linked dominant epilepsy locus causing aberrant cerebral cortical development. Neuron 1996;16:77-87

17. Fox JW, Lamperti ED, Eksioglu YZ, et al. Mutations in filamin 1 prevent migration of cerebral cortical neurons in human periventricular heterotopia. Neuron 1998;21:1315-25

18. Sheen VL, Basel-Vanagaite L, Goodman J, et al. Etiological heterogeneity of familial periventricular heterotopia and hydrocephalus. Brain Dev 2004;26:326-34

19. Barkovich AJ. Pediatric Neuroimaging. Philadelphia: Lippincott Williams \& Wilkins; 2005:8:659

20. Robertson SP, Twigg SR, Sutherland-Smith AJ, et al. Localized mutations in the gene encoding the cytoskeletal protein Filamin A cause diverse malformations in humans. Nat Genet 2003;33:487-91

21. Van Essen D. A tension-based theory of morphogenesis and compact wiring in the CNS. Nature 1997;385:313-18

22. Rakic P. Neuroscience: genetic control of cortical convolutions. Science 2004;303:1983-84

23. Piao X, Hill R, Bodell A, et al. G protein-coupled receptor-dependent development of human frontal cortex. Science 2004;303:2033-36

24. Régis J, Mangin J, Ochiai T, et al. "Sulcal root" generic model: a hypothesis to overcome the variability of the human cortex folding patterns. Neurol Med Chir (Tokyo) 2005;45:1-17

25. Caviness VS Jr. Mechanical model of brain convolutional development. Science 1975;189:18-21
26. Toro R, Burnod Y. A morphogenetic model for the development of cortical convolutions. Cereb Cortex 2005;15:1900-13

27. Goldman-Rakic P, Rakic P. Experimental modification of gyral patterns. In: Geschwind N, Galaburda A, eds. Cerebral Dominance. Cambridge, Massachusetts: Harvard University Press; 1984:179-92

28. Van Essen D, Dierker D. On navigating the human cerebral cortex response to "In Praise of Tedious Anatomy." Neuroimage 2007;37: $1050-68$

29. Cendes F, Li L, Andermann F, et al. Dual pathology and its clinical relevance. Adv Neurol 1999;81:153-64

30. Salanova V, Markand O, Worth R. Temporal lobe epilepsy: analysis of patients with dual pathology. Acta Neurol Scand 2004;109:126-31

31. Tortori-Donati P, Rossi A. Congenital malformations in the neonate. In: Rutherford M, ed. MRI of the Neonatal Brain. London, UK: Harcourt Publishers Limited; 2002:225-49

32. Andersen P, Morris R, Amaral D. The Hippocampus Book. Princeton, New Jersey: Oxford University Press USA; 2006:49

33. Margariti P, Blekas K, Katxioti F, et al. Magnetization transfer ratio and volumetric analysis of the brain in macrocephalic patients with neurofibromatosis type 1. Eur Radiol 2007;17:433-38

34. Kivitie-Kallio S, Autti T, Salonen O, et al. MRI of the brain in the Cohen syndrome: a relatively large corpus call in patients with mental retardation and microcephaly. Neuropediatrics 1998;29: 298-301

35. Lerman-Sagie T, Ben-Sira L, Achiron R, et al. Thick fetal corpus callosum: an ominous sign? Ultrasound Obstet Gynecol 2009;34: $55-61$

36. Katz MJ, Lasek RJ, Siler J. Ontophyletics of the nervous system: development of the corpus callosum and evolution of axons tracts. Proc Natl Acad Sci U S A 1983;80:5936-40

37. Kostovic I, Rakic P. Developmental history of the transient subplate zone in the visual and somatosensory cortex of the macaque monkey and human brain. J Comp Neurol 1990;297:411-70

38. Ternier J, Wray A, Puget $S$, et al. Tectal plate lesions in children. J Neurosurg 2006;104(6 suppl):369-76

39. Mitchell L, Thomas $\mathrm{P}$, Zacharin M, et al. Ectopic posterior pituitary lobe and periventricular heterotopia: cerebral malformations with the same underlying mechanism? AJNR Am J Neuroradiol 2002;23: $1475-81$

40. Guerrini R, Mei D, Sisodiya S, et al. Germline and mosaic mutations of FLN1 in men with periventricular heterotopia. Neurol 2004;13: $51-56$

41. Solé G, Coupry I, Rooryck C, et al. Bilateral periventricular nodular heterotopia in France: frequency of mutations in FLNA, phenotypic heterogeneity and spectrum of mutations. J Neurol Neurosurg Psychiatry 2009;80:1394-98

42. Hannan AJ, Servotte S, Katsnelson A, et al. Characterization of nodular neuronal heterotopia in children. Brain 1999;122:219-38

43. Battaglia G, Chiapparini L, Fraceschetti S, et al. Periventricular nodular heterotopia: classification, epileptic history, and genesis of epileptic discharges. Epilepsia 2006;47:86-97

44. Archer JS, Abbott DF, Masterton RA, et al. Functional MRI interactions between dysplastic nodules and overlying cortex in periventricular nodular heterotopia. Epilepsy Behav 2010;19:631-34 